\title{
PITOT TUBE BLOCKAGE BY MUD-DAUBER WASP AS A MECHANISM OF A MOTION OF AN AIRCRAFT THROUGH MIRCE FUNCTIONABILITY FIELD
}

\section{Jezdimir Knezevic* \\ MIRCE Akademy}

MIRCE Mechanics is the discipline of MIRCE Science that focuses on the scientific understanding and description of the phenomena that govern the motion of functionable system types though the MIRCE Functionability field [1]. A full understanding of the mechanisms that generate the motion is essential for the accurate predictions of the functionability performance of functionable system types using MIRCE Science. According to the 2nd Axiom of MIRCE Science the motion of a functionable system type through MIRCE Space is a result of imposed natural phenomena or human activities, which are jointly called functionability actions. Thus, the main objective of this paper is to address pitot tube blockage by mud-dauber wasps as a mechanism that influences the motion of an aircraft through the MIRCE Functionability Field. Although it is not a frequent and globally realised phenomenon, it is a physically observable one, which is experienced by aircraft on the ground in areas where these types of insects are present. The paper also presents a set of a possible prevention and management actions regarding this specific phenomena.

Key words: Functionability, Motion

\section{INTRODUCTION}

MIRCE Mechanics is the discipline of MIRCE Science that focuses on the scientific understanding and description of the physical phenomena and human rules that govern the motion of functionable system types though the MIRCE Functionability Field ${ }^{1}$. A full understanding of the mechanisms that generate this motion is essential for the accurate predictions of the functionability performance of a functionable system type using the mathematical scheme of MIRCE Science. [1]

On 21 November 2013, after a flight from Singapore, Etihad Airways Airbus A330, registered A6-EYJ, landed at Brisbane (Australia) airport and was taxied to the terminal. Two hours and three minutes later, the aircraft was pushed-back from the gate for the return flight to Singapore. After observing airspeed indication failure on his display the captain rejected the initial take-off attempt. The aircraft taxied back to the terminal where a troubleshooting task was carried out, before being released back into service. During the second take-off roll, the crew became aware of an airspeed discrepancy after the V1 decision speed and the take-off was continued. Once airborne, the crew declared a Mayday and decided to return to Brisbane where an overweight landing was carried out. [2]

Since 2008, four planes have returned to the airport after takeoff resulting from the same physical mechanism, with an additional three aborted takeoffs. In total, the Australian Transport Safety Bureau reported at least 15 scheduled flights were interrupted by the same physical mechanism on flights taking off from Brisbane Airport from 2008-2018 and more incidents in the previous years.

The main objective of this paper is to address the Pitot tube blockage by the mud-dauber wasp as a mechanism of the motion of the A6-EYJ through the MIRCE Functionability Field and the consequential functionability actions taken by humans in an attempt to continue with the scheduled flights. The paper also presents a set of a possible prevention and management actions regarding this specific phenomena.

\section{MIRCE SCIENCE FUNDAMENTALS}

According to the 2nd Axiom of MIRCE Science ${ }^{2}$ the motion of a functionable system type through the MIRCE Functionability Field is a result of imposed natural phenomena or human activities, which are jointly called functionability actions [1]. At any instant of calendar time, a given functionable system type could be in one of the following two observable functionability states:

- Positive Functionability State (PFS), a generic name for a state in which a functionable system type is able to deliver the expected measurable function(s),

${ }^{1}$ MIRCE Functionability Field is an infinite set of possible functionability points, each representing functionability states that a functionable system type could be found during the any instant of the calendar time [1]. It is defined by the following set:

$\operatorname{MFF}_{S}(t)=\left\{\operatorname{PFS}_{S}^{i-1}(t), N F S_{S}^{i}(t), i=1,2, ., \infty, t \geq 0\right\}$

${ }^{2}$ MIRCE Science comprises axioms, laws, mathematical equations and calculation methods that enable accurate predictions of the functionability performance of a given "future" system to be calculated [1] 
- Negative Functionability State (NFS), a generic name for a state in which a functionable system type is unable to deliver the expected measurable function(s), resulting from any reasons whatsoever.

The sequential motion of a functionable system type through the functionability states, in the direction of calendar time, is generated by functionability actions, which are classified as:

- Positive Functionability Action (PFA), a generic name for any natural process or human activity that compels a system to move to a PFS,

- Negative Functionability Action (NFA), a generic name for any natural process or human activity that compels a system to move to a NFS.

To scientifically understand the mechanisms that generate functionability events, positive and negative, analysis of the in-service behaviour of several thousands of components, modules and assemblies of functionable systems in defence, aerospace, nuclear, transportation, motorsport, communication and other industries have been conducted at MIRCE Akademy, by members of staff, students and science fellows.

In MIRCE Science all negative functionability actions are categorised as following [1]:

- Component-internal actions that consist of:

- Inherent actions that are introduced into components prior to their introduction into service through the activities associated with the design, manufacturing, handling, transportation, maintenance, storage and similar processes.

- Cumulative continuous actions that are an inevitable part of the components in-service life resulting from natural decay processes such as: corrosion, fatigue, creep, wear and similar.

- Component-external actions, which are originated by:

- Environmental phenomena that cause discrete overload, like foreign object damage; birds strike (domestic and wild animals), weather (hail, rain, snow, lightening, solar radiation, etc.,) and so forth.

- Human activities:

- Errors that are related to phenomena that cause overload, for example use and abuse by operators, (pilots, driver and other users), maintainers (maintenance induced errors) and logistics support personnel (bogus parts, shelf life, etc.)

- Rules that are related to organisational policies, legal requirements, national and international, best practices or any other human imposed functionability related actions (scheduled and condition based maintenance tasks).

- System-internal actions: resulting from processes that are taking place within a system, like a change from passive to active state for certain components and modules, a change in functionability states of some of its constituent components that impact the functionability of the system.

- System-external actions: which are generated by:

- Discrete environmental phenomena related to weather (hail, rain, snow, lightening, volcanic eruptions, wind, fog, solar radiation, etc.) and other causes that impact on the functionability of a functionable system type.

- Human activities:

- Errors, which are related to the phenomena of use and abuse by: operators, maintainers or supply chain personnel.

- Rules, which are related to organisational policies, legal requirements, national and international, best practices or any other human imposed functionability actions that cause the occurrence of NFEs for the functionable systems.

This paper discusses the Pitot tube blockage by mud-dauber wasps, as one of many component-external actions generated mechanisms, that govern a motion of an aircraft from a PFS to a NFS of the MIRCE Functionability Field and the consequential functionability actions taken by humans to return an aircraft into subsequent PFS state.

\section{PITOT STATIC SYSTEM}

In aviation, a pitot-static system is used to determine an aircraft's: airspeed, Mach number, altitude, and altitude trend. It consists of a pitot tube, a static port, and the pitot-static instruments. Other instruments that might be connected are: air data computers, flight data recorders, altitude encoders, cabin pressurisation controllers, and various airspeed switches.

Errors in the pitot-static system readings can be extremely dangerous as the information obtained from the pitot static system, such as altitude, is potentially safety-critical. Several commercial airline disasters have been traced to a failure of the pitot-static system.

\section{Pitot Tube ${ }^{3}$}

The Pitot probe consists of a tube pointing directly into the air flow and is used in measuring the stagnation pressure called total pressure or Pitot pressure (PT). It is the sum of the static and kinetic pressures and is detected as the flowing stream impacts on the pitot opening. To measure impact pressure, most pitot tubes use a small, sometimes L-shaped tube, with the opening directly facing the oncoming airflow (from the perspective of normal forward movement of the aircraft).

${ }^{3}$ Henri Pitot, in 1732, invented Pitot tubes to measure the velocity of a flowing liquid or air 
The point velocity of approach (VP) can be calculated by taking the square root of the difference between the total pressure and the static pressure (PS) and multiplying that by the C/D ratio, where $C$ is a dimensional constant and $D$ is air density:

$$
V p=\frac{\mathrm{C}}{\mathrm{D}} \sqrt{\left(P_{T}-P_{S}\right)}
$$

The flow rate is obtained by multiplying the point velocity by the cross-sectional area of the pipe or duct. It is critical that the velocity measurement be made at an insertion depth which corresponds to the average velocity. As the flow velocity rises, the velocity profile in the pipe changes from laminar to turbulent.

Static pressure is obtained through a static port, which is most often a flush-mounted hole on the fuselage of an aircraft, and is located where it can access the airflow in a relatively undisturbed area. Some aircraft may have a single static port, while others may have more than one. In situations where an aircraft has more than one static port, there is usually one located on each side of the fuselage. With this positioning, an average pressure can be taken that allows for more accurate readings in specific flight situations. A pitot-static tube effectively integrates the static ports into the pitot probe. It incorporates a second coaxial tube (or tubes) with pressure sampling holes on the sides of the probe, outside the direct airflow, to measure the static pressure. When the aircraft climbs, static pressure will decrease.

The Pitot tube is most often located on the wing or front section of an aircraft, facing forward, where its opening is exposed to the relative wind. By situating the Pitot tube in such a location, the ram air pressure is more accurately measured since it will be less distorted by the aircraft's structure.

\section{Airspeed indicator}

The airspeed indicator is connected to both the pitot and static pressure sources. The difference between the pitot pressure and the static pressure is called dynamic pressure. The greater the dynamic pressure, the higher the airspeed reported.

A traditional mechanical airspeed indicator contains a pressure diaphragm that is connected to the Pitot tube. The case around the diaphragm is airtight and is vented to the static port. The higher the speed, the higher the ram pressure, the more pressure exerted on the diaphragm, and the larger the needle movement through the mechanical linkage.

\section{Altimeter}

The pressure altimeter, also known as the barometric altimeter, is used to determine changes in air pressure that occur as the aircraft's altitude changes. Pressure altimeters must be calibrated prior to flight to register the pressure as an altitude above sea level.

The instrument case of the altimeter is airtight and has a vent to the static port. Inside the instrument, there is a sealed aneroid barometer. As pressure in the case decreases, the internal barometer expands, which is mechanically translated into a determination of altitude. The reverse is true when descending from higher to lower altitudes.

\section{Machmeter}

Aircraft designed to operate at transonic or supersonic speeds will incorporate a Machmeter. The Machmeter is used to show the ratio of true airspeed in relation to the speed of sound.

Most supersonic aircraft are limited as to the maximum Mach number they can fly, which is known as the "Mach limit". The Mach number is displayed on a Machmeter as a decimal fraction.

\section{Variometer}

The variometer, also known as the vertical speed indicator (VSI) or the vertical velocity indicator (VVI), is the pitot-static instrument used to determine whether or not an aircraft is flying in level flight.

\section{AIRSPEED MEASUREMENT ON AIRBUS A330}

The A330 has three independent systems for calculating and displaying airspeed information: (1) captain, (2) first officer, and (3) standby systems. All three located on the underside of the fuselage near the nose. Each of them has its own pitot probe, static ports, air data modules (ADMs), air data inertial reference unit (ADIRU), and airspeed indicator. Each ADIRU comprises two parts, an air data reference (ADR) part and an inertial reference (IR) part which are integrated into a single unit. One part can be switched off while the other part can still operate.

Airspeed is measured by comparing total air pressure $(\mathrm{Pt})$ and static air pressure (Ps). On the A330, this is measured using a pitot probe, and Ps is measured using two static ports. A separate ADM is connected to each pitot probe and each static port, and it converts the air pressure from the probe or port into digital electronic signals.

Each pitot probe consisted of a tube that projected several centimetres out from the fuselage, with the opening of the tube pointed forward into the airflow. The tube has drain holes to remove moisture, and it is electrically heated to prevent ice accumulation during flight.

Normally, the airspeed displayed to the captain uses the captain's pitot probe and ADIRU 1, but the source can be manually switched by the crew to the standby system (standby pitot probe and ADIRU 3) if required. Similarly, the airspeed displayed to the first officer (FO) normally uses the first officer's pitot probe and ADIRU 2, but the source can be manually switched by the crew to the standby system if required. 


\section{FLIGHT CONTROL SYSTEM ON AIRBUS A330}

The Airbus A330 has fly-by-wire flight controls, which means that the aircraft's flight control surfaces are electrically controlled and hydraulically activated in accordance with the flight control computers that process pilot and autopilot inputs to direct the movements of the control surfaces.

A330 has three flight control primary computers (FCPCs) and two flight control secondary computers (FCSCs) that continuously monitored outputs from the three ADIRUs. The median (voted) value of each parameter is compared to each individual value. If the difference is above a predetermined threshold for a predetermined confirmation time, then the associated part of that ADIRU (IR or ADR) is rejected and the two remaining sources are used for flight control purposes.

The flight control system operates according to normal, alternate or direct control laws. Under normal law, the computers prevent the exceedance of a predefined safe flight envelope. If various types of aircraft system problems are detected the control law reverted to alternate law, under which some of the protections were not provided or were provided with alternate logic. Under direct law, no protections are provided and control surface deflection is proportional to sidestick and pedal movement by the flight crew.

\section{AIRCRAFT MAINTENANCE MANUAL PARKING PROCEDURE FOR AIRBUS A330}

Parking procedures for Airbus A330 is described in the Aircraft Maintenance Manual (AMM) requests that approved protective covers are installed on each of the air data probes or devices, including Pitot probes. However, experience teaches us that many operators do not apply the AMM parking procedure, if the aircraft only has a short turn-around time or remains on the flight line.

Using the approved Pitot probe covers is important as the covers for other manufacturer's aircraft may not be the correct fit or offer complete protection for the Pitot probes of Airbus aircraft. The same Pitot probe cover can be used on Airbus A310, A320, A330, and A340 aircraft families. Airbus $A 380$ and A350 aircraft have Multi-Function Probes (MFP) and a standby Pitot probe that use two different covers. Pitot probe and MFP covers are part of the flight kit for each aircraft.

It is necessary to point out that protective covers can be installed 30 minutes after engines shut down as the probe heating is deactivated when engines are turned off. After a period of 15 minutes for the probe tip to cool to $70^{\circ} \mathrm{C}$, it can take an additional 15 minutes to reach ambient temperature.

\section{THE OBSERVED MOTION OF A6-EYJ THROUGH THE MIRCE FUNCTIONABILITY FIELD}

The sequence of observed functionability events that took place during attempting take off by Etihad Airways Airbus A330, from Brisbane (Australia) to Singapore on 21 November 2013, is briefly presented below.

\section{First Negative Functionability Action}

A red "speed flag" display on the avionics, as the aircraft accelerated through $50 \mathrm{kt}\left(50 \mathrm{knots}^{4}\right)$ on the first takeoff attempt, initiated the functionability action. [2]

According to the standard operating procedures (SOP), the captain rejected the takeoff and in doing so, compelled the change of functionability state of the aircraft, from positive to negative.

\section{Positive Functionability Actions after the First Rejected Take-off}

To return the aircraft into a PFS, maintenance engineer performed the troubleshooting procedures for an 'ADIRU1 fault message, in accordance to the Airbus provided Troubleshooting Manual (TSM). The aircraft maintenance engineer also performed a Built-In Test Equipment (BITE) test of the Electronic Flight Control System (EFCS) 1 and 2. The units tested with normal indications and no faults were identified. The TSM procedure did not specifically identify the Pitot probe as a possible cause.

Although no 'hard' (permanent) faults had been identified, the maintenance engineer, in consultation with the Operator's Maintenance Control Centre, considered that the best resolution would be to make ADIRU 1 inoperative. However, this was not permitted under the Minimum Equipment List (MEL) requirements for Extended Operation (ETOPS) dispatch. Hence, the engineer exchanged ADIRU 1 and 2 and performed a BITE test of both units. The aircraft was dispatched with the ADR part of ADIRU 2 inoperative (switched off) in accordance with the MEL. The flying officer's air data source was switched to ADIRU 3 and the captain's air data source remained switched to the normal (ADIRU 1) position. As a result of this positive functionability action the aircraft have been returned to a PFS and ready for the second take off.

\section{Second Negative Functionability Action}

The airworthy declared A330 was cleared for departure. However, during the second takeoff run the captain's airspeed indicators failed again, but this time at a speed where the SOPs call for continuing the takeoff. The Australian Transport Safety Bureau (ATSB) questioned the captain's recollection that the airspeed failed after "V1" (151 kt.), the speed at which crews are advised to continue the takeoff, noting that the flight data recorder information showed that the failure flag should have appeared after reaching $50 \mathrm{kt}$. [2]

\footnotetext{
${ }^{4}$ The knot is a unit of speed equal to one nautical mile per hour. [exactly $1.852 \mathrm{~km} / \mathrm{h}$ (approximately $1.15078 \mathrm{mph}$ )]
} 
The incorrect captain airspeed associated with ADIRU 2 inoperative caused the A330's auto-thrust and flight directors to disengage, the flight controls mode reverted from normal law to alternate law (for 8 seconds) then back to normal law (4 seconds) and finally back to alternate law. The second reversion to alternate law was latched for the remainder of the flight, which meant that the autopilot was unavailable. The pilots declared an emergency and landed at Brisbane at an aircraft weight of approximately 200 metric tons, which was 18 metric tons heavier that the A330s maximum landing weight.

\section{Positive Functionability Actions after the Second NFE}

The captain's probe was removed from the aircraft and sent to the probe manufacturer in the US for examination. In consultation with the participants in the investigation, a test plan was developed prior to examination and testing of the probe.

The probe had been on the aircraft since new (just over 7 years of continuous operation). Its condition was consistent with its time-in-service with the probe inlet showing wear, but within the component maintenance manual (CMM) limits. Visual inspection showed that there was no evidence of obstruction of the drain holes. A borescope examination was performed through the pitot inlet and also through the pneumatic port. The examination showed that the interior of the probe was occluded by an incomplete insect's nest and the nest material was consistent with that of the mud-dauber wasp. Compressed air was applied to the probe and none of the material was dislodged. The base of the nest was broken away with a sharp instrument and was fully removed by flushing with hot water.

After removal of the obstruction, the probe was tested and, according to the CMM, it could be re-certified and returned to in service use. [2]

\section{MUD-DAUBER WASPS (SPHECIDAE)}

Solitary wasps differ from the social wasps in nesting habits and life cycle. As they do not have any workers, the queens care for their own young. Hence, they usually only have a single nest. After building cells within the nest, the female wasp captures several spiders. The captured prey are stung and paralysed before being placed in the nest (usually 6-15 per nest), and then a single egg is deposited on the prey within each cell. The wasp then seals the cell with a thick mud plug. After finishing a series of cells, she leaves and does not return. The larva spins a cocoon and pupates. Eventually, the hatching larva will eat the prey and emerge from the nest. They have a low reproductive rate.

The Mud-dauber wasps ${ }^{5}$ are medium to large sized and are either shiny black or metallic blue-black with slender abdomens. They get their name from building their nests out of mud balls that are collected at puddle and pool edges. The finger-like nests are attached to flat or vertical surfaces. The mud is moulded into cells by the wasps' mandibles. Their nests are usually built in sheds, barns and other structures.

This species is found in a wide variety of habitats, such as rock ledges, man-made structures, puddles and other water edges, cypress domes, in long leaf pines and in turkey oaks.

Mud-dauber wasps are widespread in Canada, the United States, Central America, South Africa and the West Indies, and has been introduced to many Pacific Islands (including Australia, Hawaii and Japan), Peru and Europe, where it has become established in some countries of the western Mediterranean Basin (Austria, Croatia, France, Italy, Cyprus, Ukraine).

\section{PITOT TUBE BLOCKAGE HAZARD MANAGEMENT AT BRISBANE AIRPORT}

Mud-dauber wasp activity at Brisbane Airport has been investigated previously by the ATSB15 and continuing reports and incidents indicate that it is an ongoing hazard. As the wasps cannot be completely eradicated, it is necessary to have control measures in place to minimise the chance of a pitot probe becoming obstructed. [2]

Following the incident addressed in this paper, the Brisbane Airport Corporation (BAC) reviewed their Wildlife Hazard Management Plan (which includes wasp activity). The relevant part of management activities related to Pitot tube blockage by mud-dauber wasp is presented below:

- The aircraft operator has changed its policy on the use of pitot covers. They are now required to be used on all transits at Brisbane Airport, regardless of ground time.

- The aircraft manufacturer has amended its maintenance troubleshooting manual to increase the likelihood that a blocked pitot probe will be detected.

- The airport operator has extended its wasp inspection and eradication program and reviewed and updated its Wildlife Hazard Management Plan.

- In addition, $\mathrm{CASA}^{6}$ has drawn attention to the safety implications of mud wasp activity through several publications.

Reporting any occurrences of Pitot probe obstruction to the local airport authorities and Airbus will help to monitor for adverse trends, put specific measures in place and communicate this information to the benefit of all airlines and operators

\footnotetext{
${ }_{5}^{5}$ https://animalcorner.co.uk/animals/mud-dauber-wasp/

${ }^{6}$ Civil Aviation Safety Authority (CASA) is the Australian national authority for the regulation of civil aviation
} 


\section{CONCLUSIONS}

The main objective of this paper is to address pitot rube blockage by mud-dauber wasps as a physically observed mechanism of the motion of an aircraft through the MIRCE Functionability Field.

Although not a frequently occurring phenomenon, it has been experienced by several aircraft on the ground in areas where these types of insects are present. Even further in a few occasions it caused fatalities. For example, all 189 people on board died of Birgenair Flight 301 from Puerto Plata in the Dominican Republic to Frankfurt, Germany, on 6 February 1996. The B757-200 operating the route crashed shortly after take-off. The cause of crash was pilot error after receiving incorrect airspeed information from one of the pitot tubes, which investigators believe was blocked by a wasp nest built inside it. The aircraft had been sitting unused for two days without the Pitot tube covers in place. [3]

The paper has clearly demonstrated that airlines and operators should assess and monitor the risk of any obstruction to their aircraft's Pitot probes at the airports where they are based or operating to. Airports should an active role by collaborating with their operators to manage airport hazards and communicate on any of the mitigations in place.

Finally, where there is an identified risk of Pitot obstruction due to sand, dirt, dust or insect nesting activity, the operator should be obliged applying a specific policy to use Pitot covers for aircraft on the ground regardless of the lengths of turn-around times.

\section{REFERENCES}

1. Knezevic, J., The Origin of MIRCE Science, pp. 232. MIRCE Science, Exeter, UK, 2017, ISBN 978-1904848-06-6

2. Australian Transport Safety Bureau, Transport Safety Report, Air data system failure involving Airbus A330-243 A6-EYJ, pp. 24, Aviation Occurrence Investigation AO-2013-212- 6, Final 6 May 2016.

3. "Reporte Final Accidente Aereo Birgenair, Vuelo ALW-301, Febrero 06,1996" (PDF) (in Spanish). DGAC. 25 October 1996. Archived (PDF) from the original on 4 September 2014. Retrieved 27 August 2014. 